\title{
UN EXEMPLE DE PHÉNOMÉNOLOGIE MINIMALISTE: LA DISCORDANCE DES TEMPS
}

Dominique Janicaud

SINTESE - Trata-se de delimitar uma abordagem não-metafísica de uma fenomenologia minimalista do tempo, através de um reexame da concepção de temporalidade, pela desmistificação do tempo linear e pelo exercício do que seria uma discordância dos tempos.

PALAVRAS-CHAVE - Fenomenologia do tempo. Tempo linear. Temporalidade.
ABSTRACT - The article sets out to devise a nonmetaphysical approach to a minimalist phenomenology of time, by recasting a conception of temporality, through the demystification of linear time and through what would be a discord of times.

KEY WORDS - Linear time. Phenomenology of time. Temporality.

Si je n'ai pas l'intention de proposer ici une philosophie du temps, c'est évidemment parce que ce programme risque d'être excessivement ambitieux (et métaphysique), mais c'est surtout parce que je crois qu'une méthode plus modeste, exclusivement phénoménologique, peut encore porter des fruits sur le terrain de la temporalité, après Husserl, après Merleau-Ponty, après Heidegger, mais à condition que ses objectifs et ses moyens méthodologiques soient clairement définis et délimités. Je ne reviendrai pas ici en détail sur les principes du projet d'une "phénoménologie minimaliste" dont j'ai esquissé ailleurs le programme. 'Je voudrais seulement en donner un exemple ou, si l'on préfère, un modeste échantillon.

Le regard philosophique que je voudrais poser sur la temporalité consistera à montrer qu'il faut se déprendre de la conception du temps unique et linéaire, conception qui règne en Occident depuis quelques siècles, mais qui nous masque la richesse des temps et leurs discordances. Il ne faut point partir en quête du cœur du temps, car il n' y en a pas!

Université de Nice, France.

1 Voir Dominique JANICAUD, La Phénoménologie éclatée, Paris, Éditions de l'Éclat, 1998 (en particulier, le chapitre 5, "Pour une phénoménologie minimaliste").

\begin{tabular}{|l|l|l|l|l|l}
\hline VERITAS & Porto Alegre & v. 47 & n. 1 & Março 2002 & p. 13-19 \\
\hline
\end{tabular}


Pourquoi lancer l'expression "discordance des temps"? Ce titre peut déconcerter, dans la mesure où il renverse (ou contredit) une expression grammaticale consacrée. Repartons de cette expression (peut-être devenue presque inintelligible, car rares sont actuellement les connaisseurs en matière de grammaire): elle peut avoir un sens pour nous, parce que la langue française comporte l'ambiguité entre le temps grammatical et le temps comme tel. La discordance des temps est la règle qui veut que le temps du verbe de la proposition subordonnée s'accorde avec celui de la proposition principale. Cette règle - souvent mal mise en œuvren'est d'ailleurs pas mécanique: son application dépend du sens. ${ }^{2}$

Le désir -plus ou moins justifié - de faire s'accorder les temps grammaticaux au sein d'une phrase reflète un désir plus profond ou plus général d'articuler une expérience temporelle qui ne cesse d'être brisée, dissymétrique, douloureuse. Cette aspiration à la concordance n'existerait pas, s'il n'y avait pas discordance. s'il n'y avait pas une déstabilisation constamment provoquée par le temps (Aristote l'a dit: le temps est essentiellement déstabilisant).

Il ne fait pas de doute que psychologiquement, éthiquement (et sans doute déjà physiologiquement) nous avons besoin de trouver - sinon une harmonie complète - du moins un niveau d'étiage ou d'équilibre entre les trois grandes orientations temporelles. Découvrir chaque instant dans son absolue nouveauté et en sa radicale singularité peut être source d'angoisse. A l'inverse, la temporalité serait idéalement maîtrisée par une mémoire souveraine, maniée souplement, disponible pour le présent et pour l'avenir: une parfaite concordance temporelle! Notre navigation quotidienne dessine son sillage entre cette disjonction pathologique et cette harmonie fictive, trop idéale.

A partir d'observations simples, tirées de la vie quotidienne et de l'histoire de la mesure du temps, je voudrais en premier lieu tenter une démystification du temps linéaire (et des représentations abstraites ou techniques de ce temps), en second lieu ébaucher une petite phénoménologie de la discordance des temps.

\section{Démystification du temps linéaire}

Le temps est-il un long fleuve plus ou moins tranquille, laissant uniformément glisser son cours, divisible en instants tous semblables? Le temps lui-même existe-t-il de cette façon comme une forme a priori de notre perception, c'est-à-dire une forme préexistant à notre expérience et la conditionnant univoquement? Selon cette conception (qui correspond à la physique newtonienne), cette forme du temps que nous découvrons en nous (en notre "sens interne") est fixe et détient une vérité constante, au-delà des variations subjectives: ce qui est temporel (à savoir dans le temps) s'écoule, mais le temps lui-même demeure.

Telle est exactement la conception de Kant: le temps est "une forme pure de l'intuition sensible. Différents temps ne sont que des parties du même temps...".

Voir Maurice GREVISSE, Le bon usage, Paris, Geuthner, 1961, § 1047.

KANT, Critique de la raison pure, trad. Alain RENAUT, Paris, Aubier, 1997, p. 127. 
C'est "une représentation nécessaire qui joue le rôle de fondement pour toutes les intuitions". ${ }^{4}$ Et ce temps n'a qu'une dimension: celle de la succession (de ses parties).

Même si Kant repense et reformule cette théorie dans le cadre d'une théorie philosophique plus générale portant sur la connaissance, cette définition du temps correspond à celle de Newton: "Le temps absolu, vrai et mathématique, sans relation à rien d'extérieur, coule uniformément et s'appelle durée". ${ }^{5}$ Soulignons: il y a un temps vrai, sans relation à rien d'extérieur, et qui coule uniformément.

Devons-nous admettre comme définitive cette conception d'un temps unique, vrai et absolu? La science contemporaine elle-même a fait exploser ce cadre rigide, à la fois avec la découverte de la relativité de la mesure du temps par rapport à une constante universelle, la vitesse de la lumière, et avec un extraordinaire affinement des temporalités au niveau cosmologique, comme inversement au niveau subatomique.

Mais je voudrais montrer que - même sans faire intervenir ces divers dépassements (ou ces complexifications) de la physique newtonienne -, une observation attentive de notre expérience temporelle doit nous conduire à une conception pluraliste du temps.

Avant de quitter Newton et Kant, remarquons qu'il ne faut pas caricaturer leurs positions. Ainsi Newton n'ignore-t-il pas toute relativité du temps, puisqu'à côté du temps absolu il distingue "le temps relatif, apparent et vulgaire, [et] cette mesure sensible et externe d'une partie de durée quelconque" (mois, jours, heures). Pour lui, cette relativité trahit une imperfection par rapport au temps unique, vrai, en soi. Pour nous, ce sera l'inverse: les mesures seront de plus en plus précises, mais il n'y aura plus de temps unique, en soi (la relativité gagne la temporalisation elle-même).

La représentation du temps unique et linéaire nous est devenue si familière, elle a été si intériorisée, si intégrée à notre expérience mentale régulière, que nous la considérons toute naturelle et que nous nous y retrouvons assez à l'aise, car cette représentation newtonienne et kantienne reprend la vieille image du temps qui coule, en lui donnant une forme abstraite et mathématique. Vouloir la démystifier ne revient pas à prétendre qu'elle soit fausse (à l'intérieur de nos cadres spatio-temporels ordinaires) ni qu'elle ne soit pas efficace (elle a permis la maitrise collective du temps, l'acquisition de la précision dans les mesures techniques, les transports, la vie sociale -une rationalisation de l'économie et du travail) ; je soutiens seulement que cette représentation d'un temps unique et uniforme n'est pas à penser comme première ni ultime, dès lors qu'on se soucie du sens même de la temporalité dans l'expérience humaine.

Id., ibid., p. 126.

Isaac NEWTON, Principes mathématiques de la philosophie naturelle, trad. de la Marquise du CHASTELLET, reprod. de l'éd. de 1756, Paris, Blanchard, 1966, t. I, définition VIII, Scolie. 
Cette représentation du temps linéaire et uniforme est abstraite et n'a été acquise que tardivement par l'humanité. Elle n'est première ni ethno-historiquement, ni ontogénétiquement, ni phénoménologiquement.

De multiples témoignages ethnographiques ${ }^{6}$ prouvent que, si la découverte de la durée est immémoriale, elle s'est effectuée très progressivement à partir de l'observation de cycles (astraux, saisonniers, agricoles, etc.). Dans le monde occidental, la temporalité uniformément quantifiée ne s'élabore que lentement au cours du Moyen-Age à partir d'une réglementation de la vie monastique, mais le régulateur à oscillations et l'échappement mécanique sont connus dès l'an 1000 .

Ontogénétiquement, c'est-à-dire quant au développement de l'individu, Piaget a montré que la conscience de la succession se construit chez l'enfant très progressivement avant sept ans. On ne s'élève pas d'un seul coup au temps linéaire, mais par une structuration progressive, liée à la coordination des mouvements en groupements d'ordre (sériation des événements) et emboîtement (synchronisation et addition des durées). ${ }^{8}$

Enfin, une observation phénoménologique des constantes de notre vie psychologique permet de redécouvrir combien la discordance est le facteur décisif de la prise de concience temporelle. Hegel l'a montré au début de sa Phénoménologie de l'esprit en isolant la "visée du ceci", conscience prétendûment immédiate qui dit: "Maintenant, c'est la nuit" et qui découvre, quelques heures plus tard, que: "Maintenant, c'est le jour". L'identité du "maintenant" est d'autant plus claire et affirmée qu'elle a dû traverser la différenciation et le changement. Chaque maintenant est différent d'un autre -et pourtant j'arrive à dégager l'unité du "maintenant" comme tel: c'est tout le paradoxe de l'expérience temporelle. Illustrons cette discordance qui me paraît constituer le ressort de cette expérience.

\section{Petite phénoménologie de la discordance des temps}

Le rôle de la discordance peut tout d'abord être mis en évidence par l'observation psychologique de l'attention au temps et l'observation plus générale du rôle de la monstration et des marques, qui constituent autant de repères pour l'élaboration des mesures temporelles.

C'est une observation courante, très ancienne, mais aisément renouvelable (par exemple, dans une salle d'attente): le songe, la distraction, une tâche absorbante font oublier le temps; à l'inverse, l'attente, l'obsession chronologique font trouver le temps interminable, voire insupportable. L'attention est une condition

\footnotetext{
6 Voir, en particulier, Maurice LEENHARDT, Do Kamo, Paris, Gallimard, 1971; E.E. EVANSPRITCHARD, Les Nuer, Paris, Gallimard, 1994.

7 Voir David LANDES, L'heure qu'il est, Paris, Gallimard, 1987, p. 95: "L'Europe n'a pas été la seule à construire des machines compliquées, mais la seule à le faire en abstrayant la fonction temps de son mécanisme".

8 Voir Jean PIAGET, Le développement de la notion de temps chez l'enfant, Paris, PUF, 1946.
} 
essentielle de la perception du temps; ${ }^{9}$ nous n'avons pas conscience du temps dans le sommeil. A la limite, ne pas s'occuper de l'heure ni du temps, les laisser dans l'indifférence en supprime l'existence, au moins au niveau psychologique des effets du temps. Au contraire, il est évident que l'obsession du temps en renforce encore la cruauté.

Plus généralement, moins psychologiquement, il n'y a pas mesure du temps sans marqueur. Robinson dans son île doit reconstituer un calendrier, en inscrivant des repères sur un arbre, sans quoi les jours glissent indifféremment. Les premières mesures pratiques du temps ont été obtenues en répétant des écoulements ou des combustions identiques (le sablier, la clepsydre, la chandelle étalonnée). Pour mesurer le temps, il faut au préalable en isoler une unité, marquer un rythme, délimiter une durée.

La temporalité ne livre d'elle-même que son fait brut: il y a du temps. Même ce fait n'a pu se livrer que parce qu'un différer a été observé ou isolé. Montrer (en révélant une telle observation), c'est temporaliser: qu'il s'agisse d'un instant ou d'un durée plus longue. ${ }^{10}$

Il y a discordance du temps non seulement dans l'inévitable dissociation entre passé, présent et futur (quelle que soit la dimensiion qu'on accentue), mais de nouveau au sein de chacune des trois dimensions.

Ainsi les "palais de la mémoire" (expression de Saint Augustin) sont-ils d'une richesse que nous ne soupçonnons pas (car notre mémoire comporte une part immense d'inconscient). Mais surtout le rappel de tel souvenir déterminé ne s'effectue jamais tout à fait de la même façon et n'épuise nullement le contexte frémissant d'où l'on extrait cet îlot de lumière. Merleau-Ponty l'a montré après Bergson: la manière dont je convoque un souvenir modifie à chaque fois l'intensité, la tonalité, parfois même le sens de ce souvenir. Fragilité de notre mémoire? A coup sûr ; mais cette fragilité tient à sa constitution même: n'étant pas un spectateur extérieur et intemporel de mon passé, je bouge, je m'enrichis moimême à chaque instant de nouvelles impressions, pensées, expériences, de telle sorte qu'un même événement passé (mon mariage, tel accident, telle rencontre) est sans cesse recontextualisé, réinterprété, relu à la lumière de ce que je deviens. La passé n'est jamais le même ; il n'est pas figé ; il a eu un futur vierge; et nous lui surimposons des futurs eux-mêmes dépassés, mais aussi ce futur neuf qui entame notre présent. C'est pourquoi ce temps grammatical particulièrement riche - le futur antérieur - permet de conjurer (dans une certaine mesure) cette discordance.

Il faut perdre le temps pour le retrouver, mais on ne le retrouve jamais comme on l'avait perdu. Nous en avons un exemple saisissant chez Proust. ${ }^{11}$ L'apparition

\footnotetext{
- ARISTOTE, Physique, IV, 219 a: "Nous disons que du temps s'est passé, quand nous percevons de l'antérieur et du postérieur dans le mouvement".

${ }^{10}$ Peut-être est-ce l'origine du mot "montre" et du Zeiger en allemand, l'aiguille (littéralement le "montreur").

1 Nous reprenons, dans ce paragraphe, quelques lignes d'un texte paru dans la Revue d'esthétique: "La phénoménologie sur les traces de l'inapparence du temps" (n. 36, 1999, p. 25-36).
} 
des clochers de Martinville, soudain alignés grâce à la mobilité de la voiture a-telle donné au narrateur un plaisir qu'il essaie de restituer en son récit, tout en sachant cet essai presque impossible, puisqu'il insère dans le cours principal du récit un bref extrait qu'il avait écrit dans la voiture elle-même, comme pour capter l'insaisissable, parce qu'il ressentait en sa merveilleuse fugacité l'impression de l'alignement des clochers dans le soleil couchant. ${ }^{12}$ En lisant ces pages de Proust, nous participons à notre tour à cette joie créatrice, ou plutôt à la genèse recréatrice de la complexité quasi musicale de nouveautés intentionnelles jouant dans la mémoire elle-même et offrant non une perspective définitive sur le passé, mais -grâce aux dégradés de l'écriture - ce que Merleau-Ponty nomme "la coexistence de présents incompossibles". ${ }^{13}$

Un tel essai pour décrire et penser de manière "minimaliste" ${ }^{14}$ l'articulation entre la visibilité et l'invisibilité du temps représente une ambition évidemment plus modeste et plus limitée que les grandes philosophies du temps. Il ne s'agit plus d'atteindre l'essence du temps (ni l'originaire-essentiel de la temporalité), mais de porter l'attention sur le jeu des temporalisations, sans méconnaître la difficulté de saisir, de voir et même de dire les processus temporels.

Tel celui du futur et du présent, le coeur du passé est toujours discordant et cette discordance ne se livre à nous jamais purement, mais à travers le réseau enchevêtré et renouvelé d'intentionnalités brisées qu'il faut traverser et traduire en mots, comme le suggère Proust: "ce qui était caché derrière les clochers de Martinville devait être quelque chose d'analogue à une jolie phrase, puisque c'était sous la forme de mots qui me faisaient plaisir que cela m'était apparu". ${ }^{15}$

En conclusion, le temps linéaire et uniforme masque trop souvent les discordances très riches, mais aussi douloureuses, qui font l'inépuisable diversité des expériences temporelles. Ces discordances répparaissent aujourd'hui, aussi bien dans l'explosion techno-scientifique des mesures du temps que dans la redécouverte psychologique, phénoménologique et historique du feuilleté des temporalités. Par exemple, la "nouvelle histoire" (cette école historique française inspirée par les travaux de Fernand Braudel autour de la revue des Annales) ne se réfère plus à l'unique chronologie des événements politiques et diplomatiques, mais démultiplie les temporalités, à différents niveaux (chronologies longues des évolutions climatiques, des cycles économiques et même de l'évolution des mentalités).

Cependant, la difficulté ne s'arrête pas là. Il ne suffit pas de mettre de côté le temps linéaire, pour autant que ce soit possible. En fait, ce n'est pas si facile, puisque notre vie quotidienne, professionnelle, technique et même nos loisirs sont soumis à l'obsession chronométrique. Une discordance inédite et subtile réside dans le fait que l'uniformisation du temps et son homogénéisation fonctionnelle

\footnotetext{
Voir Marcel PROUST, A la recherche du temps perdu, Bibliothèque de la Pléiade, I, p. 180-182. Maurice MERLEAU-PONTY, Notes de cours (1959-1961), Paris, Gallimard, 1996, op. cit., p. 207. Dans mon livre Chronos, Paris, Grasset, 1997.

PROUST, op. cit., p. 181.
} 
créent des nuisances psycho-sociales nouvelles (dont le décalage horaire des vols intercontinentaux est un exemple emblématique).

Cette complexification de nos expériences temporelles n'est pas mauvaise en soi. Nous ne sommes pourtant pas toujours capables d'y faire face. La question du temps ouvre ainsi sur la question de l'art de vivre: comment trouver et appliquer une éthique de la réinvention des temps? L'expression "avoir du cœur à l'ouvrage" est significative: les discordances du temps exigent de nous autant de réponses, c'est-à-dire - en termes temporels - autant de rythmes, qui ne doivent pas être imposés de l'extérieur, de manière purement technique. S'il y a un remède à la discordance des temps, il ne peut se trouver sans cœur. 